\title{
A NOTE ON AMALGAMS OF INVERSE SEMIGROUPS
}

\author{
BENJAMIN STEINBERG
}

(Received 22 November 1999; revised 28 June 2000)

Communicated by D. Easdown

\begin{abstract}
This note gives a necessary condition, in terms of graded actions, for an inverse semigroup to be a full amalgam. Under a mild additional hypothesis, the condition becomes sufficient.

2000 Mathematics subject classification: primary $20 \mathrm{M} 18$.

Keywords and phrases: Inverse semigroups, amalgams, graded actions.

This note introduces graded actions of inverse semigroups, a notion already implicit in the work of Lawson on ordered representations [2] and the author on semidirect products of inverse semigroups [7]. If one studies inverse semigroups from the inductive groupoid point-of-view (see for instance [3]), then these are exactly the sorts of actions which arise from inductive groupoid actions as per the author's [7]. Subsequent work of the author and Lawson [4] will show that graded partial actions of inverse semigroups lead to a natural proof of the structure theorem for idempotent pure extensions of inverse semigroups [3]. In this paper, we use graded actions to provide a generalization of the 'ping-pong' theorem for amalgamated products of groups [5, Proposition 12.4] to full amalgams of inverse semigroups. Namely, we give a condition for an inverse monoid to be a full amalgam and show that essentially all full amalgams arise in this way. We assume some basic familiarity with inverse semigroups [3].
\end{abstract}

\section{Graded actions}

If $I$ is an inverse monoid, we use $E(I)$ for the set of idempotents of $I$. Viewing $E(I)$ as a partially ordered set, via the natural partial order, we let, for $e \in E(I)$,

The author was supported in part by NSF-NATO postdoctoral fellowship DGE-9972697 and by FCT through Centro de Matemática da Universidade do Porto.

(C) 2001 Australian Mathematical Society 0263-6115/2001 \$A2.00+0.00 
$[e]=\{f \in E(I) \mid f \leq e\}$. If $X$ is a set, we use $I(X)$ to denote the inverse monoid of all partial bijections of $X$ (acting on the left); that is, the monoid of all bijections between subsets of $X$ with composition of relations. An action of an inverse semigroup $I$ on $X$ is then a homomorphism $\varphi: I \rightarrow I(X)$. Normally, we write $m x$ for $\varphi(m)(x)$ ( $m \in I, x \in X$ ). If $I$ is an inverse monoid, the action is called unital if $\varphi(1)=1$. We say the action is graded if there exists a function $p: X \rightarrow E(I)$ such that

(i) $\operatorname{dom}(\varphi(e))=p^{-1}([e])$;

(ii) if $t^{-1} t=p(x)$, then $t t^{-1}=p(t x)$.

We call $p$ the grading. Observe that any (unital) action of a group is graded. If only condition (i) holds, we call the action weakly graded. Weakly graded actions will be enough to prove our main theorem, but from the point-of-view of inductive groupoids, graded actions are more natural.

PROPOSITION 1.1. Let I have a weakly graded action on $X$ via $\varphi$ with grading $p$. Then for $t \in I, \operatorname{dom}(\varphi(t))=p^{-1}\left(\left[t^{-1} t\right]\right)$ and $\operatorname{ran}(\varphi(t))=p^{-1}\left(\left[t t^{-1}\right]\right)$.

PROOF. By considering inverses, it suffices to deal with the domain of $\varphi(t)$. For $f \in I(X), \operatorname{dom}(f)=\operatorname{dom}\left(f^{-1} f\right)$ and so

$$
\operatorname{dom}(\varphi(t))=\operatorname{dom}\left(\varphi\left(t^{-1} t\right)\right)=p^{-1}\left(\left[t^{-1} t\right]\right)
$$

as desired.

To show that graded actions are natural, in fact, prevalent, we point out that the Preston-Wagner representation [3] of an inverse semigroup is graded. The action is given by $\varphi: S \rightarrow I(S)$ with $\varphi(s): s^{-1} s S \rightarrow s s^{-1} S$ given by $\varphi(s)(t)=s t$. If one defines $p: S \rightarrow E(S)$ by $p(s)=s s^{-1}$, then it is not difficult to see that $p$ is a grading for $\varphi$.

\section{Full amalgams of inverse semigroups}

If $I$ is an inverse semigroup and $e \in E(I)$, we will write $I_{e}$ for the $\mathscr{H}$-class of $e$. If $T$ is an inverse subsemigroup of $I$, we say that $T$ is a full subsemigroup if $E(T)=E(I)$. If $I_{1}$ and $I_{2}$ are inverse semigroups with $I_{1} \cap I_{2}=T$, then the amalgam $I_{1} *_{T} I_{2}$ is the inverse semigroup with the usual universal property. The amalgam is called full if $T$ is a full subsemigroup of both $I_{1}$ and $I_{2}$. The following result is a reformulation of [1, Theorem 2] which is, in turn, a reformulation of the main theorem of [6]. 
THEOREM 2.1. Let $S$ be an inverse semigroup generated by full inverse subsemigroups $S_{1}$ and $S_{2}$ and let $T=S_{1} \cap S_{2}$. Then $S \cong S_{1} *_{T} S_{2}$ if and only if given a product $s=a_{1} \cdots a_{n} t$ with the $a_{j}$ alternately in $S_{1} \backslash T$ and $S_{2} \backslash T, a_{j}^{-1} a_{j}=a_{j+1} a_{j+1}^{-1}$, $a_{n}^{-1} a_{n}=t t^{-1}, t \in T$, and with $t \notin E(S)$ if $n=0$, one has $s \notin E(S)$.

We now give a condition for an inverse semigroup to be an amalgam in terms of weakly graded actions. We use $\subset$ to denote strict containment.

THEOREM 2.2. Let $S$ be an inverse semigroup generated by full inverse subsemigroups $S_{1}$ and $S_{2}$ with $S_{1} \cap S_{2}=T$ such that there exists $i \in\{1,2\}$ with $\left[\left(S_{i}\right)_{e}: T_{e}\right]>2$ for all $e \in E(S)$. Let $S$ have a weakly graded action on a set $X$ with grading $p$ and let $X_{1}$ and $X_{2}$ be disjoint non-empty subsets of $X$. Suppose further:

(i) $\left(S_{1} \backslash T\right) X_{1} \subseteq X_{2},\left(S_{2} \backslash T\right) X_{2} \subseteq X_{1}$;

(ii) $T X_{1} \subseteq X_{1}, T X_{2} \subseteq X_{2}$;

(iii) for $e \in E(S), p^{-1}([e]) \cap X_{1}$ and $p^{-1}([e]) \cap X_{2}$ are both non-empty.

Then $S=S_{1} *_{T} S_{2}$.

PROOF. We imitate the proof of [5, Proposition 12.4]. Suppose $s=a_{1} \cdots a_{n} t \in$ $E(S)$ with the $a_{j}$ alternately in $S_{1} \backslash T$ and $S_{2} \backslash T, n>0, t \in T, a_{j}^{-1} a_{j}=a_{j+1} a_{j+1}^{-1}$, and $a_{n}^{-1} a_{n}=t t^{-1}$; we obtain a contradiction. Without loss of generality, we may assume that $a_{n} \in S_{1}$. For $e \in E(S)$, let $X_{i, e}=X_{i} \cap p^{-1}([e]), i=1,2$. By assumption, for all $e \in E(S), X_{i, e} \neq \emptyset, i=1,2$. Also, by Proposition 1.1 and from conditions (i) and (ii), it follows that if $r \in S_{1} \backslash T$, then $r X_{1, r^{-1} r} \subseteq X_{2, r^{-1}}$ (and dually for $r \in S_{2} \backslash T$ ) and if $r \in T$, then $r X_{i, r^{-1} r} \subseteq X_{i, r r^{-1}}, i=1,2$. Observe that $s=s^{-1} s=t^{-1} t$ and $s=s s^{-1}=a_{1} a_{1}^{-1}$. Thus, since $s \in E(S)$ must act as a partial identity and

$$
s X_{1, s}=a_{1} \cdots a_{n} t X_{1, r^{-1} t},
$$

we see that $n=2 k$ for some $k>0$.

We claim, for $i=1, \ldots, n-1$,

$$
a_{i} a_{i+1} X_{1, a_{i+1}^{-1} a_{i+1}} \subset X_{1, a_{i} a_{i}^{-1}}
$$

where $a_{i+1} \in S_{1} \backslash T$ (and hence $a_{i} \in S_{2} \backslash T$ ). Our above observations show that $a_{i} a_{i+1} X_{1, a_{i+1}^{-1} a_{i+1}} \subseteq X_{1, a_{i} a_{i}^{-1}}$. We must now show that this containment is strict. First suppose that, for all $e \in E(S),\left[\left(S_{1}\right)_{e}: T_{e}\right]>2$. Let $e=a_{i+1}^{-1} a_{i+1}$ and choose $r, u \in\left(S_{1}\right)_{e} \backslash T_{e}$ such that $r u^{-1} \notin T_{e}$. Then it follows that at least one of $a_{i+1} r$ and $a_{i+1} u$ is not in $T$; say $h=a_{i+1} r \notin T$. Note that $h^{-1} a_{i+1}=r^{-1} e=r^{-1} \in S_{1} \backslash T$, $h^{-1} h=r^{-1} e r=e$, and $h h^{-1}=a_{i+1} r r^{-1} a_{i+1}^{-1}=a_{i+1} a_{i+1}^{-1}$. So $h^{-1} a_{i+1} X_{1, e} \subseteq X_{2, e}$ whence $a_{i+1} X_{1, e} \subseteq h X_{2, e}$. Thus

$$
a_{i+1} X_{1, e} \cap h X_{1, e} \subseteq h X_{2, e} \cap h X_{1, e}=\emptyset .
$$


Since $X_{1, e} \neq \emptyset, h^{-1} h=e=a_{i+1}^{-1} a_{i+1}$, and $h h^{-1}=a_{i+1} a_{i+1}^{-1}$, it follows, from condition (iii) and Proposition 1.1, that

$$
\emptyset \neq h X_{1, e} \subseteq X_{2, a_{i+1} a_{i+1}^{-1}}
$$

and so

$$
a_{i+1} X_{1, a_{i+1}^{-1} a_{i+1}} \subset X_{2, a_{i+1} a_{i+1}^{-1}}
$$

It now follows, since $a_{i}^{-1} a_{i}=a_{i+1} a_{i+1}^{-1}$, that $a_{i} a_{i+1} X_{1, a_{i+1}^{-1} a_{i+1}} \subset X_{1, a_{i} a_{i}^{-1}}$ A similar argument shows that if, for all $e \in E(S),\left[\left(S_{2}\right)_{e}: T_{e}\right]>2$, then $a_{i} X_{2, a_{i}^{-1} a_{i}} \subset X_{1, a_{i} a_{i}^{-1}}$ and the claim follows.

From the above claim and since $t X_{1, s} \subseteq X_{1, s}$, it follows that $s X_{1, s} \subset X_{1, s}$ contradicting $s$ being an idempotent.

We now prove a strong converse to the above theorem. Let $T$ be a full inverse subsemigroup of an inverse semigroup $S$. For $s, s^{\prime} \in S$ with $s \mathscr{R} s^{\prime}$, one says that $s \sim_{L} s^{\prime}$ if $s^{-1} s^{\prime} \in T$. It is not hard to see that this is an equivalence relation and that if $a^{-1} a=s s^{-1}$, then $s \sim_{L} s^{\prime}$ implies as $\sim_{L} a s^{\prime}$. By a complete set of left coset representatives of $T$ in $S$, we mean a complete set of representatives for $\sim_{L}$. Note that, for $t, t^{\prime} \in T$ with $t \mathscr{R} t^{\prime}, t \sim_{L} t^{\prime}$.

THEOREM 2.3. Suppose $S_{1}, S_{2}$ are inverse semigroups with $T=S_{1} \cap S_{2}$ a full subsemigroup of $S_{1}$ and $S_{2}$. Then there exists a graded action of $S_{1} *_{T} S_{2}$ on a set $X$ with grading $p$ and disjoint non-empty subsets $X_{1}$ and $X_{2}$ such that

(i) $\left(S_{1} \backslash T\right) X_{1} \subseteq X_{2},\left(S_{2} \backslash T\right) X_{2} \subseteq X_{1}$;

(ii) $T X_{1} \subseteq X_{1}, T X_{2} \subseteq X_{2}$;

(iii) for $e \in E(S), p^{-1}([e]) \cap X_{1}$ and $p^{-1}([e]) \cap X_{2}$ are both non-empty.

ProOF. Let $S=S_{1} *_{T} S_{2}$ and consider the Preston-Wagner representation of $S$; we saw earlier that this action is graded. Choose a complete set of left coset representatives of $T$ in $S$. Then it is shown in [1], using the results of [6], that each element $s \in S$ has a unique factorization of the form $s=a_{1} \cdots a_{n} t$ with the $a_{j}$ left coset representatives, alternately in $S_{1} \backslash T$ and $S_{2} \backslash T ; t \in T ; a_{j}^{-1} a_{j}=a_{j+1} a_{j+1}^{-1}$; and $a_{n}^{-1} a_{n}=t t^{-1}$. Let $X_{1}$ be the collection of elements of $S$ whose factorizations begin with an element of $S_{2} \backslash T$ and $X_{2}$ the collection of elements whose factorizations begin with an element of $S_{1} \backslash T$. It is straightforward to verify that, for the Preston-Wagner representation of $S, X_{1}$ and $X_{2}$ have the desired properties.

\section{References}

[1] S. Haataja, S. W. Margolis and J. Meakin, 'Bass-Serre theory for groupoids and the structure of full regular semigroup amalgams', J. Algebra 183 (1996), 38-54. 
[2] M. V. Lawson, 'A class of actions of inverse semigroups', J. Algebra 179 (1996), 570-598.

[3] _ Inverse semigroups: The theory of partial symmetries (World Scientific, Singapore, 1999).

[4] M. V. Lawson and B. Steinberg, 'Partial actions of inverse semigroups and idempotent pure homomorphisms', in preparation.

[5] R. C. Lyndon and P. E. Schupp, Combinatorial group theory (Springer, Berlin, 1977).

[6] K. S. S. Nambooripad and F. J. Pastijn, 'Amalgamation of regular semigroups', Houston J. Math. 15 (1989), 249-254.

[7] B. Steinberg, 'Factorization theorems for morphisms of ordered groupoids and inverse semigroups', Proc. Edinburgh Math. Soc., to appear.

Faculdade de Ciências

da Universidade do Porto

4099-002 Porto

Portugal

e-mail: bsteinbg@agc0.fc.up.pt 